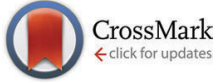

Cite this: Phys. Chem. Chem. Phys., 2016, 18, 1292

Received 9th October 2015, Accepted 24th November 2015

DOI: $10.1039 / c 5 c p 06084 b$

www.rsc.org/pccp

\section{Micro- and nano-tubules built from loosely and tightly rolled up thin sheets $\dagger$}

\author{
Luisa Losensky, ${ }^{a}$ Björn Goldenbogen, ${ }^{b}$ Gudrun Holland, ${ }^{c}$ Michael Laue, ${ }^{c}$ \\ Anca Petran, ${ }^{d}$ Jürgen Liebscher, ${ }^{d}$ Holger A. Scheidt, ${ }^{e}$ Alexander Vogel, \\ Daniel Huster, ${ }^{e}$ Edda Klipp ${ }^{b}$ and Anna Arbuzova*a
}

\begin{abstract}
Tubular structures built from amphiphilic molecules are of interest for nano-sensing, drug delivery, and structuring of oils. In this study, we characterized the tubules built in aqueous suspensions of a cholesteryl nucleoside conjugate, cholesterylaminouridine (CholAU) and phosphatidylcholines (PCs). In mixtures with unsaturated PCs having chain lengths comparable to the length of CholAU, two different types of tubular structures were observed; nano- and micro-tubules had average diameters in the ranges 50-300 $\mathrm{nm}$ and 2-3 $\mu \mathrm{m}$, respectively. Using cryo scanning electron microscopy (cryo-SEM) we found that nano- and micro-tubules differed in their morphology: the nano-tubules were densely packed, whereas micro-tubules consisted of loosely rolled undulated lamellas. Atomic force microscopy (AFM) revealed that the nano-tubules were built from 4 to $5 \mathrm{~nm}$ thick CholAU-rich bilayers, which were in the crystalline state. Solid-state ${ }^{2} \mathrm{H}$ NMR spectroscopy also confirmed that about $25 \%$ of the total CholAU, being about the fraction of CholAU composing the tubules, formed the rigid crystalline phase. We found that CholAU/PC tubules can be functionalized by molecules inserted into lipid bilayers and fluorescently labeled PCs and lipophilic nucleic acids inserted spontaneously into the outer layer of the tubules. The tubular structures could be loaded and cross-linked, e.g. by DNA hybrids, and, therefore, are of interest for further development, e.g. as a depot scaffold for tissue regeneration.
\end{abstract}

\section{Introduction}

Self-assembly of amphiphilic molecules into tubular structures is of great interest for biomedical and technological applications: lipid tubules have found application in inorganic nanowire and nano-tubule production, as drug delivery vehicles, and for structuring eatable oils to margarines. ${ }^{1-8}$ Many amphiphilic molecules were reported to assemble into nano-tubules structures with typical outer diameters from 10 to $1000 \mathrm{~nm}$, either flexible with single- or double-bilayer walls or rigid with multilayered walls. ${ }^{1,2,9-16}$ Sterols, which typically crystallize in water, were found to self-assemble into tubules and helices in mixtures

\footnotetext{
${ }^{a}$ Molecular Biophysics, Institute of Biology, Humboldt-Universität zu Berlin, Invalidenstr. 42, 10115 Berlin, Germany.E-mail: arbuzova@cms.hu-berlin.de

${ }^{b}$ Theoretical Biophysics, Institute of Biology, Humboldt-Universität zu Berlin, Invalidenstr. 42, 10115 Berlin, Germany

${ }^{c}$ Robert Koch Institute, ZBS 4, Seestr. 10, 13353 Berlin, Germany

${ }^{d}$ National Institute of Research and Development for Isotopic and Molecular Technologies, Donat 67-103, RO-400293 Cluj-Napoca, Romania

${ }^{e}$ Institute of Medical Physics and Biophysics, University of Leipzig, Härtelstr. 16-18, 04107 Leipzig, Germany

$\dagger$ Electronic supplementary information (ESI) available: CholAU- $d_{6}$ synthesis procedure, and additional light, electron and atomic force microscopy images. See DOI: $10.1039 / \mathrm{c} 5 \mathrm{cp} 06084 \mathrm{~b}$
}

with other lipophilic molecules, e.g. bile salts or other sterols. ${ }^{17-19}$ Uracil-appended cholesterol was found to form fibers gelating cyclohexane even at $1 \mathrm{wt} \%$ due to cholesterol-cholesterol interactions and the uracil-uracil hydrogen bonding. ${ }^{20}$ We have recently shown that another cholesteryl nucleoside conjugate, cholesterylaminouridine (CholAU, Fig. 1), self-assembled into tubular structures in the presence of an unsaturated phospholipid. ${ }^{21}$ In mixtures containing between 20 and $50 \mathrm{~mol} \%$ of CholAU, the formation of tubules with outer diameters in the nano- to micrometer ranges was observed. In mixtures containing fully or partially saturated lipids only nano-tubules were formed. ${ }^{21}$ Although the nano-tubules built in the mixtures of 30 : 70 mol\% CholAU : PC were intrinsic assemblies enriched in CholAU up to $92 \mathrm{~mol} \%$, the self-assembly process required the excess of phospholipids. ${ }^{21,22}$ As CholAU itself is practically insoluble in water, we suggested that phospholipids serve as a platform allowing the assembly. ${ }^{21,22}$ The nano-tubules were stable and could be imaged by electron microscopy after airdrying. $^{21,22}$ The micro-tubular structures were a bit less enriched in CholAU (up to $80 \mathrm{~mol} \%$ ). ${ }^{21}$ The micro-tubules were stable in aqueous solutions for years; however, they collapsed upon air-drying indicating a different morphology than usually observed for lipid nano-tubules. ${ }^{21}$ Only a few examples of micro-tubules built from amphiphilic molecules were reported: microtubules formed 

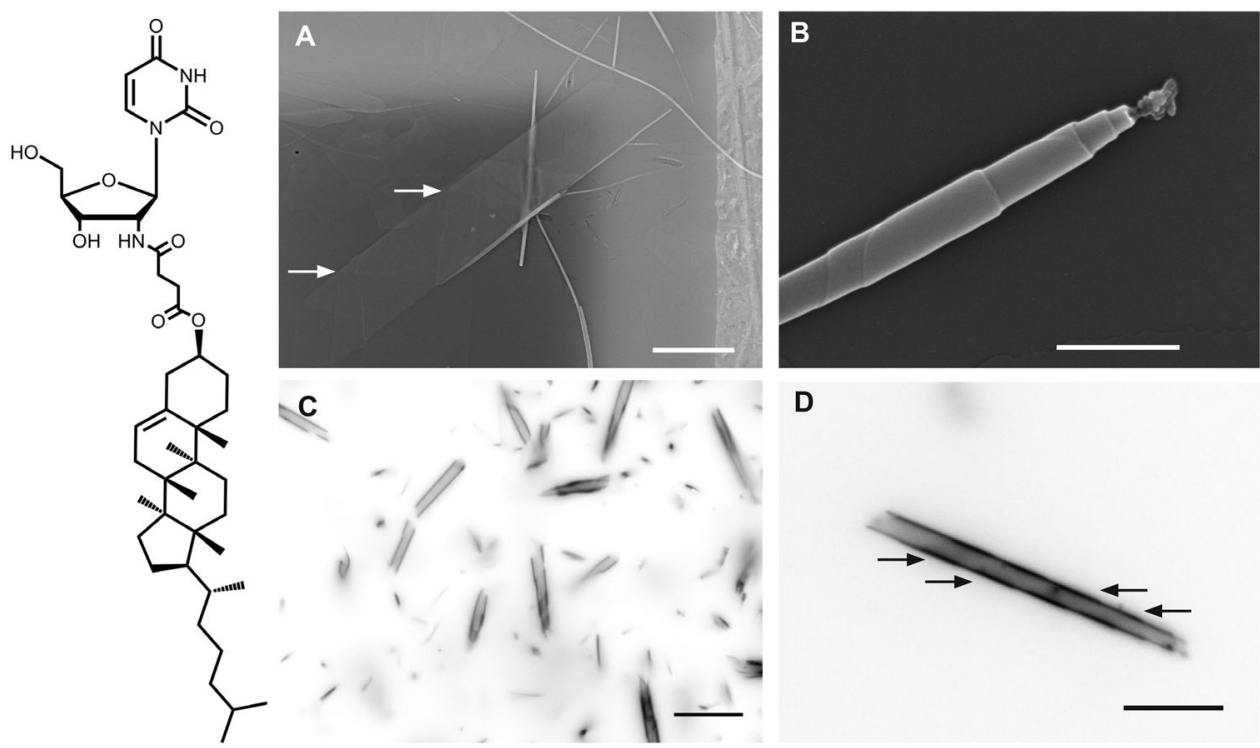

D

Fig. 1 (left) Chemical structure of 2'-N-(2-(cholesteryl)-succinyl)-2'-desoxy-2'-aminouridine (CholAU). (right) An overview of the tubular structures observed in CholAU/DMOPC samples. (A) Typical self-assembled structures, e.g. nano-tubules and flat sheets, in CholAU/DMoPC samples imaged by scanning electron microscopy (SEM). Arrows point to helical markings. Scale bar corresponds to $5 \mu \mathrm{m}$. (B) A CholAU/DMoPC nano-tubule with resolved steps on the surface due to the multilamellar morphology (SEM). Scale bar corresponds to $500 \mathrm{~nm}$. (C) The inverted fluorescence microscopy image of a 59.5 : $40: 0.5$ mol\% DMoPC: CholAU : NBD-DMPE sample revealing nano- and micro-tubules. (D) The inverted fluorescence microscopy image of a micro-tubule with helical markings indicated with arrows in another 59.5: $40: 0.5 \mathrm{~mol} \%$ DMoPC: CholAU : NBD-DMPE sample. Scale bars in (C) and (D) correspond to $10 \mu \mathrm{m}$. Note that helical markings were also observed on the flat sheets revealed by SEM, indicating that these were the collapsed microtubules (see enlarged Fig. $1 \mathrm{~A}$ in the ESI $\dagger$ ).

in bile salt solutions of cholesterol ${ }^{10}$ and from ethanolamine salt of 12-hydroxy stearic acid ${ }^{23}$ in water. Hence, here we searched for the optimal conditions for the micro-tubule formation and tested different phosphatidylcholines.

To describe the morphology of the most unperturbed tubular structures, we have now used cryo scanning electron microscopy (cryo-SEM). Schoen and colleagues used replica TEM (freeze-fracture followed by replication with metal and carbon shadowing) to visualize helical und tubular structures of diacetylenic lipids in aqueous solutions. ${ }^{14,24}$ Cryo-SEM images of multiple lipid layers and onion-like structures built from amphiphilic molecules in aqueous solutions were reported..$^{25-27}$ For example, Duerr-Auster et al. observed that polyglycerol ester of fatty acids assembled in water into multilamellar vesicles and into layers with average interlayer distances of $200-800 \mathrm{~nm}$ upon heating to $80{ }^{\circ} \mathrm{C}$ and controlled cooling to room temperature. ${ }^{25}$ DPPC was reported to build interdigitated lipid sheets when DPPC vesicles were incubated with ethanol: freeze-fracture EM revealed $\mu \mathrm{m}$-size sheets. ${ }^{28}$ Böttcher and colleagues recently summarized the cryo-TEM results on tubular structures. ${ }^{29}$

Here, we compare the self-assembled structures in binary mixtures of CholAU with phosphatidylcholines having different acyl chain lengths from 14 to 24 carbons. We report for the first time on the loose morphology of the CholAU/PC micro-tubules revealed by cryo-SEM. We have also studied the properties of the CholAU/PC nano-tubules and the lamellas building these tubules using atomic force microscopy (AFM). Furthermore, we investigated the phase state and dynamics of the deuterated
CholAU using solid-state NMR in samples containing CholAU nano-tubules.

\section{Experimental section}

\section{Materials}

1,2-Dimyristoleoyl-sn-glycero-3-phosphocholine (DMoPC, 14:1), 1,2-dipalmitoleoyl-sn-glycero-3-phosphocholine (16:1), 2-dioleoylsn-glycero-3-phosphocholine (DOPC, 18:1), 1,2-dieicosenoyl-snglycero-3-phosphocholine (20:1), 1,2-dierucoyl-sn-glycero-3-phosphocholine (22:1), 1,2-dinervonoyl-sn-glycero-3-phosphocholine (24:1), 1,2-dipalmitoyl-sn-glycero-3-phosphoethanolamine- $N$-(7-nitro-21,3-benzoxa-diazol-4-yl) ammonium salt (NBD-DPPE), and 1,2dimyristoyl-sn-glycero-3-phosphoethanolamine- $N$-(7-nitro-2-1,3benzoxadiazol-4-yl), ammonium salts (NBD-DMPE), and 1-acyl2-\{12-[(7-nitro-2-1,3-benzoxadiazol-4-yl)amino]dodecanoyl $\}-s n$-glycero3-phosphocholine (NBD-PC) were purchased from Avanti Polar Lipids (Alabaster, AL). Chemical structures of the PCs used are shown in Scheme S2 (ESI $\dagger$ ). Chloroform, cyclohexane, ethanol, and methanol were supplied by Merck (Darmstadt, Germany). Methyl- $\beta$-cyclodextrin (m $\beta \mathrm{CD}$, 1.6-2.0 mol $\mathrm{CH}_{3}$ per unit anhydroglucose) was purchased from Sigma-Aldrich (Taufkirchen, Germany). 2'- $N$-(2-(Cholesteryl)-succinyl)-2'-desoxy$2^{\prime}$-aminouridine (CholAU) was synthesized as described previously. ${ }^{21}$ CholAU- $d_{6}$ was synthesized as described in the ESI $\dagger$ (Scheme S1) using a partially deuterated precursor $\left(2,2,3,4,4,6-d_{6}\right.$ cholesterol, 97-98\%, Eurisotop, Saarbrücken, Germany). 


\section{Sample preparation}

For experiments with pure CholAU, 200-300 $\mu \mathrm{g}$ of CholAU were

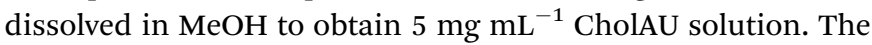
solution was warmed to $70{ }^{\circ} \mathrm{C}$ and injected using a Hamilton syringe into $1 \mathrm{~mL}$ of water at $70{ }^{\circ} \mathrm{C}$. After incubation at $70{ }^{\circ} \mathrm{C}$ for 1 hour, the suspension was cooled slowly to room temperature. For binary mixtures, $100-300 \mu \mathrm{g}$ of CholAU dissolved in chloroform/ $\mathrm{MeOH}(5: 1)$ were mixed with 300-600 $\mu \mathrm{g}$ of PCs in chloroform. The solvents were carefully evaporated under a stream of nitrogen. The resulting lipid film was dissolved in $200 \mu \mathrm{L}$ of cyclohexane/ethanol (95:5), and then the samples were frozen in liquid nitrogen and lyophilized for at least $3 \mathrm{~h}$ (Alpha 1-2 LD Plus, Martin Christ, Germany). $200 \mu \mathrm{L}$ of water $\left(70{ }^{\circ} \mathrm{C}\right)$ were added to the samples in a PCR tube. The samples were vortexed and placed either into a water bath $(10 \mathrm{~L})$ at $70{ }^{\circ} \mathrm{C}$ or into a PCR thermocycler (Bio-Rad Laboratories $\mathrm{GmbH}$, Munich, Germany) set to $71^{\circ} \mathrm{C}$ (temperature inside a PCR tube, $70{ }^{\circ} \mathrm{C}$ ). The samples were incubated for $1 \mathrm{~h}$ at $70{ }^{\circ} \mathrm{C}$, and then slowly cooled in the water bath or in the thermocycler at rates of $0.1 \mathrm{~K} \mathrm{~min}^{-1}$ to $68.5{ }^{\circ} \mathrm{C}, 0.5 \mathrm{~K} \mathrm{~min}^{-1}$ to $60{ }^{\circ} \mathrm{C}, 1 \mathrm{~K} \mathrm{~min}^{-1}$ to $50{ }^{\circ} \mathrm{C}$, and $2 \mathrm{~K} \mathrm{~min}^{-1}$ to $22^{\circ} \mathrm{C}$. Both protocols resulted in a similar yield of tubules; however, a shorter incubation at $70{ }^{\circ} \mathrm{C}(\mathrm{min})$ or a quick cooling led to the formation of shorter $(1-5 \mu \mathrm{m})$, partially folded sheets and ribbons. The samples were stored at room temperature or at $4{ }^{\circ} \mathrm{C}$. Most samples made of DMoPC and DOPC contained 0.5 mol\% NBD-DMPE and NBD-DPPE, respectively: 59.5:40:0.5 DMoPC:CholAU:NBD-DMPE and 69.5: $30: 0.5$ DOPC:CholAU: NBD-DPPE mol\% mixtures were used. The fluorescent lipids were used at this low concentration as markers to allow visualization by fluorescence microscopy; they did not influence the assembly. In binary mixtures of CholAU and DOPC without fluorescently labeled lipids the same structures were observed. ${ }^{21} 5 \mathrm{mM}$ of methyl- $\beta$-cyclodextrin $(\mathrm{m} \beta \mathrm{CD})$ were added to a sample containing nano- and microtubules of CholAU/DMoPC at room temperature.

\section{Light microscopy}

All images were taken using an inverted IX-81 fluorescence microscope (Olympus, Tokyo, Japan) and a $60 \times$ (N.A. 1.35) oilimmersion objective using U-MWNiba (BP470-495, BA520IF, DM510-550) and U-MNG2 (BP572-612, DM 562) filter sets for green and red fluorophores, respectively, and an Andor Clara interline CCD camera (Andor Technology Ltd, UK) at $25{ }^{\circ} \mathrm{C}$. Some images were inverted with 8-bit for a better visibility of the tubular structures.

\section{Transmission electron microscopy (TEM)}

For negative staining TEM a small volume $(5 \mu \mathrm{L})$ of the suspension was dispensed on the plastic film of a sample support (400 mesh copper grid, coated with Pioloform, stabilized with a thin layer of carbon and hydrophilized by glow discharge). Excess suspension was removed by gentle blotting with a wetted filter paper and the sample was stained with $0.25 \%$ aqueous uranyl acetate. The samples were analyzed using a TEM 902 (Zeiss, Oberkochen, Germany) operated at $80 \mathrm{kV}$. Micrographs were recorded using a slow scan CCD camera (Proscan, Scheuring, Germany).

\section{Scanning electron microscopy (SEM) at room temperature}

The samples were adsorbed on sample supports as described above for TEM, air-dried after blotting and then sputter-coated with approximately $2 \mathrm{~nm}$ of gold-palladium (MED 020, Leica). The samples were examined using a LEO 1530 field-emission scanning electron microscope (Zeiss, Germany) operated at $5 \mathrm{kV}$ (working distance $=5 \mathrm{~mm}$ ) using the in-lens secondaryelectron detector alone or in combination with the secondaryelectron detector according to Everhard Thornley (signal mixing).

\section{Freeze-fracture cryo-SEM (cryo-SEM)}

The samples were frozen by high-pressure freezing using a high-pressure freezer (HPF; HPF compact 01; Engineering Office M. Wohlwend GmbH, Switzerland) or the self-pressurized rapid freezing (SPRF) method. ${ }^{30}$ Both methods use high pressure to minimize ice-crystal growth in the samples but the mode of pressure generation and application is different. For HPF, two sample holders were used: (1) a platelet holder (Engineering Office M. Wohlwend GmbH, Switzerland) and (2) a tube holder (Engineering Office M. Wohlwend $\mathrm{GmbH}$, Switzerland). To use the platelet holder, a copper slot grid $(2 \times 1 \mathrm{~mm}$, Agar G2500C) was placed on a flat aluminum platelet (Engineering Office $\mathrm{M}$. Wohlwend $\mathrm{GmbH}$, Switzerland) and $1 \mu \mathrm{L}$ of suspension was added into the slot. A second flat platelet was used to cover the slot; the assembly was inserted into the sample holder and frozen under high pressure. For freezing with the tube holder, the sample suspension was filled into a copper capillary tube (0.3 mm inner and $0.45 \mathrm{~mm}$ outer diameter, Engineering Office $\mathrm{M}$. Wohlwend $\mathrm{GmbH}$, Switzerland), sealed at one end by clamping, then inserted into the tube holder, and subsequently frozen in the HPF. For SPRF, the suspension was sucked into a copper capillary tube $(0.22 \mathrm{~mm}$ inner and $0.5 \mathrm{~mm}$ outer diameter; LS404669 JV, Goodfellow, Germany), both tube ends were sealed with a claw and the sealed tubes were plunged into liquid nitrogen. ${ }^{30}$ Cryo-fixed samples were stored in liquid nitrogen until further processing. To fracture the frozen samples, the platelet sandwiches were separated mechanically, while the tubes were cut using either a cryoultramicrotome (UC7 with FC7; Leica operated at $-160{ }^{\circ} \mathrm{C}$, feed $=$ $250 \mathrm{~nm}$, cutting speed $=100 \mathrm{~mm} \mathrm{~s}^{-1}$ ) equipped with a diamond knife (cryotrim $45^{\circ}$, Diatome, Switzerland) or a tube-cuttingdevice made of steel (Engineering Office M. Wohlwend GmbH, Switzerland). The fractured samples on platelets were mounted on a particular cryo-transfer - sample holder for platelets (clamp holder for $3 \mathrm{~mm}$ specimen platelets, 16LZ04746VN, Leica). Sectioned tubes were clamped in a homemade cryo-transfer holder, which was similar to the holder developed by Bastacky et al. ${ }^{31}$ For freeze-etching and metal coating the sample holder was transferred into a pre-cooled cryo-sputter coater (MED 020, Leica), freeze-etched at $-100{ }^{\circ} \mathrm{C}$ for $5-10^{\circ} \mathrm{min}$, subsequently sputter-coated with $2 \mathrm{~nm}$ platinum at $-110{ }^{\circ} \mathrm{C}$ and finally transferred to the cooled scanning electron microscope (LEO 1530 Scanning Electron Microscope; Zeiss, Germany). All cryo-transfers were carried out using the Leica VCT-System 
(VCT 100 Shuttle and VCT 100 loading stage; Leica, Germany). The samples were examined at $-100{ }^{\circ} \mathrm{C}$ stage temperature (cold trap $=-135{ }^{\circ} \mathrm{C}$ ), at an accelerating voltage of $3 \mathrm{kV}$ (working distance $=4 \mathrm{~mm}$ ) using the in-lens secondaryelectron detector. The diameters of the tubules were analyzed using ImageJ (Rasband, W.S., NIH, Bethesda, Maryland, USA).

\section{Atomic force microscopy (AFM)}

All AFM measurements were performed in the quantitative imaging $\left(\mathrm{QI}^{\mathrm{TM}}\right)^{32}$ mode on an JPK NanoWizard ${ }^{\circledR} 3$ (JPK Instruments, Berlin, Germany) using a triangular-shaped MLCT cantilever (Bruker, Camarillo, CA, USA) with nominal spring constants of 0.1 and $0.6 \mathrm{~N} \mathrm{~m}^{-1}$. Aqueous sample suspension was dropped onto freshly cleaved mica and air dried for $10 \mathrm{~min}$. The probe was then further dried with a nitrogen flow. AFM was performed on three independent preparations of tubules. Force microscopy, first at low resolution $(64 \times 64$ pixels $)$ to identify the structures and then at high resolution $(256 \times 256$ or $512 \times$ 512 pixels) for imaging and analysis, was performed using $\mathrm{QI}^{\mathrm{TM}}$ providing an approach and a retraction force curve for each pixel. The information on the height and stiffness of the sample surface was extracted from the approach curve. The information on adhesion of the sample surface was extracted from the retraction curve. The analysis was carried out using JPK DP software, Oracle.8, and SigmaPlot 13.0. Polynomial plane fit subtraction was used to correct for the sample support tilt. The cross sections of flat structures were always parallel to the scan direction. The cross sections of tubules were always done at the top parallel to the walls of the tubules.

\section{NMR spectroscopy}

For the NMR spectroscopy, a sample containing $60 \mathrm{~mol} \%$ DMoPC, $20 \mathrm{~mol} \%$ CholAU, and $20 \mathrm{~mol} \%$ CholAU- $d_{6}(0.5 \mathrm{mg}$ of CholAU- $d_{6}$ ) was prepared in $1 \mathrm{~mL}$ of water. It was then lyophilized and rehydrated to a water content of $40 \mathrm{wt} \%$. Static ${ }^{2} \mathrm{H}$ solid-state NMR measurements were carried out on a Bruker AVANCE 750 WB NMR spectrometer (Bruker BioSpin, Rheinstetten, Germany) at a resonance frequency of $115.1 \mathrm{MHz}$ using a probe with a $5 \mathrm{~mm}$ solenoid coil at a spectral width of $\pm 250 \mathrm{kHz}$ using quadrature phase detection, a phase-cycled quadrupolar echo sequence $^{33}$ with two $3.5 \mu \mathrm{s} \pi / 2$ pulses separated by a $30 \mu \mathrm{s}$ delay. The recycle delay was $1 \mathrm{~s}$. The samples were measured at a temperature of $30{ }^{\circ} \mathrm{C}$. About 250000 scans were accumulated. ${ }^{2} \mathrm{H}$ NMR spectra were processed using a program written in Mathcad (MathSoft, Cambridge, MA). ${ }^{34}$ Typically, one NMR spectrum was accumulated per sample and the selected samples were repeated to confirm reproducibility.

\section{Results and discussion}

In agreement with our previous observations, self-assembly in binary mixtures of cholesterylaminouridine (CholAU) and dioleoylphosphocholine (DOPC, 18:1) or dimyristoleoylphosphocholine (DMoPC, 14:1) depended significantly on the molar ratio of the components. The highest yield of micrometer diameter tubules was observed in mixtures containing 30-40 mol\% of CholAU (Fig. 1). When pure CholAU predissolved in methanol was injected into water, the formation of $\mu \mathrm{m}$-sized CholAU crystals was observed. CholAU crystals resembled cholesterol crystals (Fig. S1, ESI $\dagger$ ). In mixtures with higher than $50 \mathrm{~mol} \%$ CholAU, similar crystals were observed, indicating that CholAU precipitated. When a mixture contained less than $20 \mathrm{~mol} \%$ CholAU, flexible phospholipid-characteristic structures, e.g. vesicles and tethers, ${ }^{35,36}$ were observed. Only in mixtures containing 20-50 mol\% of CholAU, both rigid tubular and phospholipid-characteristic structures were found (Fig. 1). Interested in the tubular assembly, we therefore used further mixtures containing 30-40 mol\% CholAU and 70-60 mol\% PCs.

To study a possible impact of the lipid chain length on the assembly, we compared the self-assembled structures in binary mixtures of CholAU and monounsaturated PCs with chain lengths of 14 to 24 carbons. The chemical structures of the PCs are shown in Scheme S2, ESI. $\dagger$ Note that the position of the double bond was almost the same in all tested lipids. Nanotubules were observed in all binary mixtures tested independent of the length of PC acyl chains, which indicated separation of CholAU (Fig. S2, ESI $\dagger$ ). Flat crystalline sheets were rarely seen in the aqueous solutions of the samples containing tubules; whereas helical ribbons and micro-tubules with helical markings were observed (Fig. 1C and D). Correspondingly, the flat structures observed in air-dried samples often resembled folded sheets with helical markings and were probably the collapsed micro-tubules (Fig. 1A, enlarged in ESI $\dagger$ ).

We have recently reported the formation of CholAU complexes with $\mathrm{m} \beta$-cyclodextrin $(\mathrm{m} \beta \mathrm{CD})$, known to bind cholesterol and its analogs, ${ }^{39-41}$ detected via mass spectroscopy. ${ }^{22}$ We now observed that addition of an excess of $5 \mathrm{mM} \mathrm{m} \beta \mathrm{CD}$ destroyed the micro-tubules (Fig. S3, ESI $\dagger$ ). In contrast, washing with Triton X-100 removing a significant part of phospholipids did not affect the micro-tubules. ${ }^{21}$ This indicates an essential role of CholAU for the stability of these tubular structures.

We further investigated the tubular morphology in mixtures of CholAU and DMoPC $(14: 1)$ or DOPC (18:1) using cryo scanning electron microscopy (cryo-SEM) on freeze-fractured samples. The samples were high-pressure frozen in their liquid environment without the generation of large ice crystals, which otherwise could destroy their structural organization. Highpressure freezing is considered as the gold standard fixation method for organic materials. ${ }^{42,43}$ We have used two different approaches for high-pressure freezing of sample suspensions: a high-pressure freezer or a self-pressurizing tube system. Both systems gave almost identical results and revealed all classes of tubules observed in aqueous suspension by light microscopy. However, due to the high water content of the samples, ice crystal growth was still observed around the tubules within the aqueous matrix, which was indicated by a mesh-like appearance of the matrix (Fig. 3B and C). Since the tubes appeared rather intact and only slightly undulated, we did not extend our analysis to samples mixed with cryo-protectants, e.g. dextran.

Fracturing of the samples was performed either by splitting the aluminum platelets used for high-pressure freezing of 
samples in the machine or by sectioning the copper tubes with a steel or a diamond knife, to achieve fractures through or
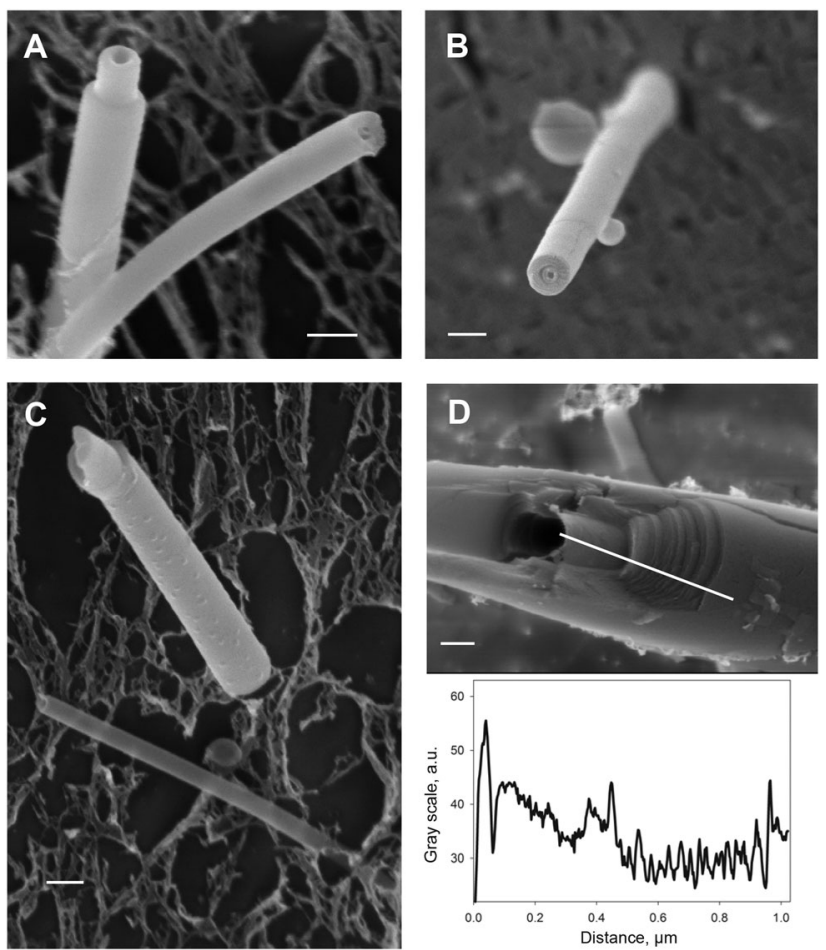

Fig. 2 Cryo-SEM images revealing densely packed nano-tubules. (A) The cryo-SEM image of CholAU/DMoPC nano-tubules with major outer diameters of 270 and $180 \mathrm{~nm}$. (B) The cryo-SEM image of CholAU/DOPC nano-tubules with outer and inner diameters of 220 and $25 \mathrm{~nm}$ and an interlayer space of about $10 \mathrm{~nm}$. (C) The cryo-SEM image of CholAU/ DMoPC nano-tubules with outer diameters of 300 and $80 \mathrm{~nm}$, respectively. (D) A medium-size tubule of CholAU/DMoPC with an outer diameter of $1 \mu \mathrm{m}$ and inner diameter of $300 \mathrm{~nm}$. At least 20 steps of densely packed lamellas can be distinguished in the fraction and in the gray level line profile shown below. The white line indicates the position of the line profile. Scale bars correspond to $200 \mathrm{~nm}$. along the tubule axis. Both methods resulted in fractured or sectioned tubules that were visible after removal of the water by freeze-etching (sublimation) the surface of the sample.

Cryo-SEM revealed nano-tubules with average outer diameters in the range 50-300 $\mathrm{nm}$ for both DOPC and DMoPC containing samples. Fig. 2A-C shows CholAU/DMoPC and CholAU/DOPC nano-tubules with major outer diameters of $270,180,300$, and $220 \mathrm{~nm}$. The lumen diameters were in the range $30-70 \mathrm{~nm}$; therefore, lamellas of the nano-tubules show high curvatures, especially the innermost layers. Note that some nano-tubules were bending during cryo-SEM investigation, presumably due to the local heating of the sample by the beam. The nano-tubules had usually densely packed walls in agreement with the conclusion based on the negative staining transmission EM (TEM) images of dry samples. The heavy metal stain used for TEM was trapped mainly in the lumen of the tubules (Fig. S4, ESI $\dagger$ ). The morphology of the nano-tubules was independent of the acyl chain length for DOPC and DMoPC.

Similar appearance of tubule profiles generated by the different methods (diamond knife, Fig. 2A; steel knife, Fig. 2B) could be explained by the fact that the sectioning was done at a rather high velocity and step size ("feed") or with a steel knife so that both rather break samples than making them plane by sectioning. However, the nano-tubules remained intact or plane in the cross-section (Fig. 2A-C), thus making it difficult to determine the number of lamellas their walls consisted of. Fig. 2D shows an example of a thicker $(\sim 1 \mu \mathrm{m}$ outer diameter) densely packed tubule of CholAU/DMoPC. The fracture revealed interconnected layers: at least 20 steps between successive layers could be resolved (Fig. 2D).

Fig. 3 illustrates the micro-tubules imaged using cryo-SEM. For the first time, the morphology of the micro-tubules was resolved: they were loosely rolled, comprising 2-6 lamellas in the walls with interlayer distances of 0-400 $\mathrm{nm}$. This explains why the micro-tubules could not be air-dried without losing their shape. Occasionally, connections (layers adhered, spacing $0 \mathrm{~nm}$ ) between the lamellas were observed. The morphology of
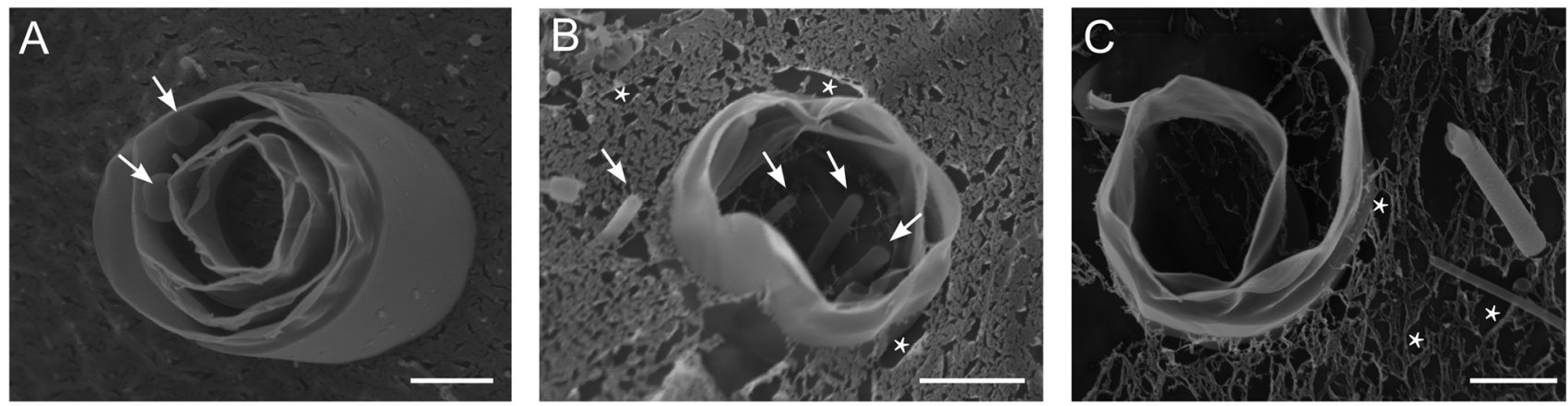

Fig. 3 Cryo-SEM images of CholAU/DOPC (A) and CholAU/DMoPC (B and C) samples. (A) A micro-tubule with an outer diameter of $2.8 \mu \mathrm{m}$ and inner diameter of 1.2-1.5 $\mu \mathrm{m}$. Three vesicles of $400-500 \mathrm{~nm}$ diameters are detected inside the tubule between the layers (arrows). Interlayer distances of $50 \mathrm{~nm}$ to $400 \mathrm{~nm}$, as well as close connections between the layers were observed. (B) A micro-tubule with outer and inner diameters of 2 and $1.8 \mu \mathrm{m}$, respectively. Three nano-tubules inside and one outside the microtubule were observed (arrows). (C) CholAU/DMoPC rolled up sheets on the left and two nano-tubules with 100 and $300 \mathrm{~nm}$ diameters on the right, respectively. The matrix in (B) and (C) reveals a mesh-like appearance with angular holes (asterisks) which is interpreted as an indication of ice crystal formation. The holes are formed during the sublimation process in which water is removed from the surface layer of the sample leaving holes where bulk water was localized. Scale bars correspond to $1 \mu \mathrm{m}$. 

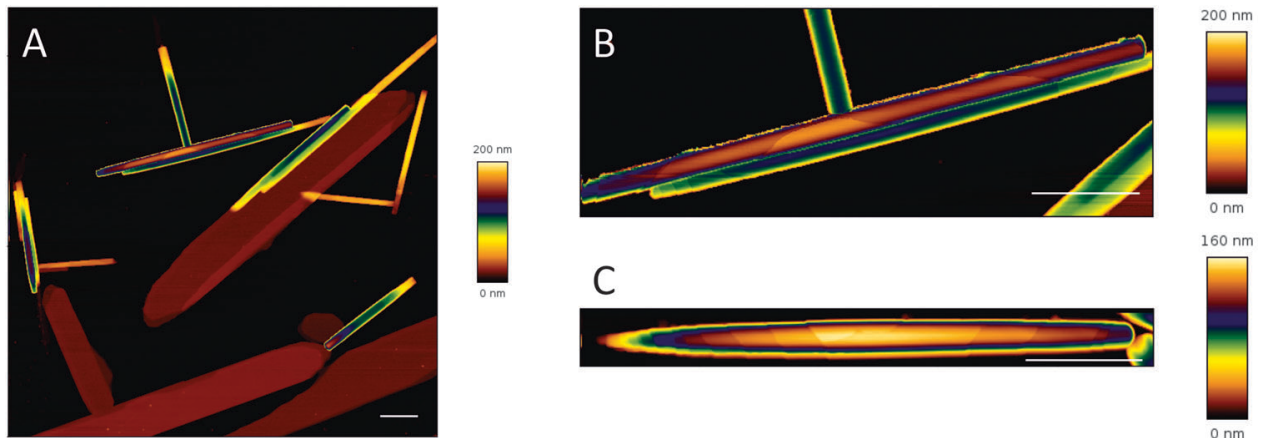

Fig. 4 AFM topography images of air-dried CholAU/DMoPC samples obtained from the AFM QI ${ }^{\mathrm{TM}}$ results. (A) An overview of the structures $(512 \times 512$ pixels). (B) Nano-tubules re-scan revealing a rolled-up structure $(512 \times 188$ pixels). (C) A nano-tubule with a clearly visible "croissants" rolled-up structure $(512 \times 51$ pixels). Scale bars represent $1 \mu \mathrm{m}$.

the micro-tubules was very similar for DOPC and DMoPC (Fig. 3A and B, respectively). Vesicles and thin densely packed tubules were sometimes found inside the lumen of the microtubules (indicated with arrows in Fig. 3A and B; see also Fig. S5-S7, ESI $\dagger$ ). The outer diameters of the micro-tubules of $2-3 \mu \mathrm{m}$ measured from cryo-SEM images were similar to the outer diameters obtained from light microscopy images for both DOPC and DMoPC containing samples. Note that not only tubular structures of various diameters, but also single lamellar sheets with non-flat topologies were observed (Fig. 3C and Fig. S6, ESI $\dagger$ ). More cryo-SEM images of tubules, curved sheets, and phospholipid-characteristic multilamellar vesicles for CholAU/DOPC and CholAU/DMoPC samples are shown in the ESI $\dagger$ (Fig. S5-S7).

Taking together, using cryo-SEM and light microscopy on aqueous samples, we have visualized analogous structures. Cryo-SEM allowed resolving for the first time the loosely rolled morphology of the micro-tubules, which was strikingly different from the morphology of the tightly rolled nano-tubules. All structures, i.e. micro-tubules, nano-tubules, sheets, and vesicles, formed in the same mixture. This indicates an inhomogeneous distribution of the components. We have reported that microand nano-tubules were enriched in CholAU up to 80 and $92 \mathrm{~mol} \%$, respectively, although the initial mixture contained in total $30 \mathrm{~mol} \%$ of CholAU. ${ }^{21}$ The excess of the phospholipids with the rest of CholAU formed phospholipid-characteristic structures, e.g. vesicles and tethers (flexible tubules with mostly unilamellar walls). Note that, as expected for unsaturated PCs, ${ }^{44}$ swelling of DOPC or DMoPC alone resulted in the formation of small and giant unilamellar and multilamellar vesicles and tethers. Rigid tubules were not observed in samples with pure DOPC or DMoPC.

We then compared the properties of the lamellas in tubular and flat structures built in CholAU/DMoPC mixtures using a force spectroscopy based imaging mode of atomic force microscopy (AFM), in which at each pixel position a complete forcedistance curve was acquired $\left(\mathrm{QI}^{\mathrm{TM}}\right) \cdot{ }^{32}$ Tubules and flat structures with total heights of about 50-200 and 4-100 nm, respectively, were observed (Fig. 4 and 5).

Fig. 4 and 5A show AFM topography images of air-dried samples prepared from $60: 40$ (mol\%) DMoPC: CholAU mixtures. Fig. 5 shows the three AFM images, e.g. height (A), adhesion (B), and stiffness (C) maps, which were obtained for each scanned region. White and black arrows point to nano-tubules and a flat structure with lipid spots on it, respectively. Note that the round spots of high adhesion and low stiffness are characteristic for phospholipids dried on mica. We zoomed into the individual structures to resolve the steps on the surface (Fig. 4B, C and Fig. S8, S9, ESI $\dagger$ ). Steps were clearly distinguishable on the surface and at the ends of the tubules. Often, the nano-tubules
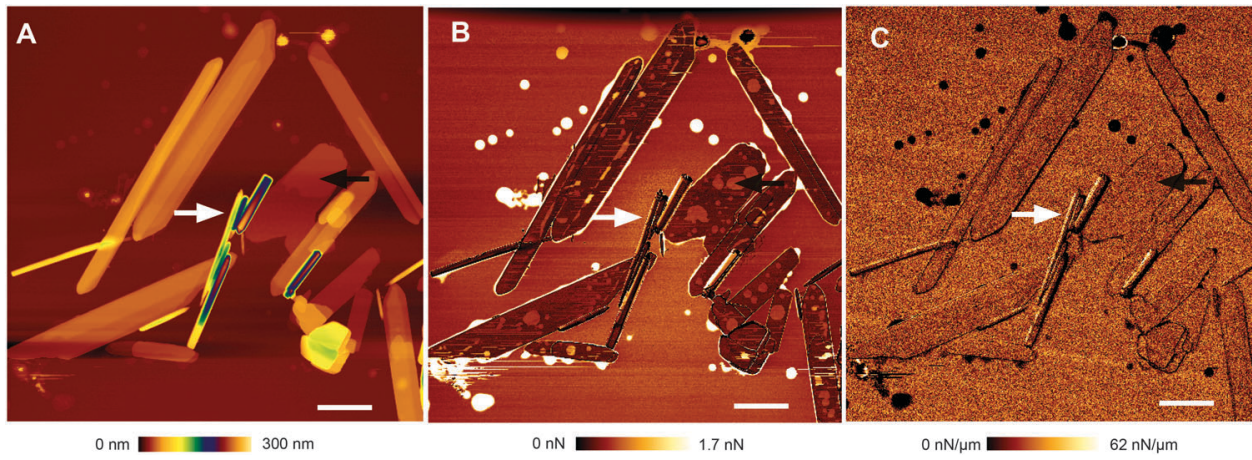

Fig. 5 Overview of the AFM images of an air-dried CholAU/DMoPC sample (512 × 512 pixels). (A) Height; (B) adhesion; (C) stiffness maps. The scales are represented by color codes. White and black arrows point to the nano-tubules and a flat structure with lipid resting on it, respectively. No difference in the slopes of the self-assembled structures and the mica support was observed. Scale bars correspond to $2 \mu \mathrm{m}$. 
resembled straight "croissants" having the highest triangle in the middle with steps going down to the ends indicating that the tubules were rolled from one sheet (Fig. 4B and C). Cross sections of the cropped topography images were analyzed to obtain the height of the steps (corresponding to the thickness of one or several lamellas, see Fig. S9, ESI $\dagger$ ). If only the smallest characteristic steps were taken into account, lamellas in the tubules were on average $4.57 \pm 0.87 \mathrm{~nm}$ thick $(n=82$, SD). Often two or four lamellas were stacked resulting in steps of 8-9 or $16 \mathrm{~nm}$ thickness ( $n=6 ; n=5$; Fig. S9, ESI $\dagger$ ).

Flat structures had usually a total thickness of about 15-100 nm with many lamellae distinguishable by steps; but sheets with heights of $4 \mathrm{~nm}(n=5)$ and $8-9 \mathrm{~nm}(n=3)$ were also observed. If only the smallest characteristic steps in flat structures were taken into account, a lamella thickness of $4.07 \pm$ $0.46 \mathrm{~nm}(n=30, \mathrm{SD})$ was obtained, which is similar to the thickness of one lamella in the tubular structures of $4.57 \pm$ $0.87 \mathrm{~nm}(n=82, \mathrm{SD})$. In addition, some residuals of lipid coating were observed on height and adhesion maps around the structures and on the top of flat structures (Fig. 5). The adhesion resulted from the attraction of the silicon nitride cantilever tip to lipid patches. It is known that phospholipids have a high affinity to freshly cleaved mica, e.g. rupture of lipid vesicles on mica is used for the formation of supported bilayers, and that air-drying destroys phospholipid bilayers. ${ }^{45,46}$

Although, AFM has a significantly better height resolution and allowed resolving the steps on the surface of the nanotubules, it might introduce edge artifacts or even change the assembly; ${ }^{47}$ therefore, it is important to note that the same morphology of nano-tubules was observed using AFM, TEM, SEM, and cryo-SEM (Fig. 2, 4 and Fig. S4, S9, S11, ESI $\dagger$ ). This also confirmed that air-drying did not change these structures significantly.

Usually, lipid tubules are formed from bilayer sheets. The lamella thickness of diacetylenic phospholipid tubules is about $6.5 \mathrm{~nm} .{ }^{48}$ The AFM height images of the diacetylenic nanotubules revealed steps of about 13-14 nm corresponding to two stacked bilayers. ${ }^{49}$ Depending on the lipid concentration and solvent mixture, Spector et al. reported controlling the wall thickness of the tubules from single- and double- to multiplebilayer thickness. ${ }^{15}$ Nano-ribbons formed in a calcium-cholate system revealed repeated strips of $2.8 \mathrm{~nm}$ - a bilayer thickness of cholate. ${ }^{50}$ Cholesterol (Chol) flat crystalline structures were composed of stacked bilayer of $3.4 \mathrm{~nm}$ thickness. ${ }^{51,52}$ The DMoPC bilayer thickness of $3.72 \pm 0.02 \mathrm{~nm}$ increased to $4.26 \pm 0.01 \mathrm{~nm}$ upon addition of $44.4 \mathrm{~mol} \%$ of $\mathrm{Chol}^{37}$ Similarly, addition of $47 \mathrm{~mol} \%$ Chol increased the thickness of fluid DMPC from 4.0 to $4.4 \mathrm{~nm} .{ }^{53,54}$ Although, the thickness of the lamellas observed by us was similar to the DMoPC/Chol bilayer thickness, the lamellas both in tubules and flat structures did not resemble the supported phospholipid bilayers. ${ }^{55}$ First, no piercing damage of the nano-tubules could be achieved even with the stiffest of the used cantilevers (nominal $k=0.6 \mathrm{~N} \mathrm{~m}^{-1}$ ), although the used cantilevers pierced easily through phosphatidylcholine bilayers (data not shown). Second, we observed that tubules were much stiffer than phospholipid bilayers: the slopes of the force-distance curves, when the tip of the cantilever was in contact with the surface, were similar for the surface of the tubules, flat structures and the surface of mica (Fig. 5). The slope of a force curve corresponds to the elastic properties of a sample, ${ }^{56}$ e.g. stiffness. Third, no lateral shifting of the lamellas was observed. Finally, the properties of the CholAU/PC lamellas were rather similar to the properties of cholesterol crystals (Fig. S11, ESI $\dagger$ ). Although the initial binary mixture contained only $30 \mathrm{~mol} \%$ of CholAU, the micro- and nano-tubules were found to be enriched in CholAU up to 80 and $92 \mathrm{~mol} \%$, respectively. We recently reported that the solubility threshold of CholAU in DMoPC bilayers is about $40 \mathrm{~mol} \% .^{22}$ Above this threshold, the segregation of CholAU into the rigid crystalline phase is expected. Similarly, the solubility limit of cholesterol in DOPC and DMPC bilayers is about 66-67 mol\%, above which the formation of cholesterol crystals was reported. ${ }^{57-59}$ Taking into account that nano-tubules consist of about $90 \mathrm{~mol} \%$ of CholAU, which is expected to segregate into the rigid crystalline phase, and only about $10 \mathrm{~mol} \%$ of phospholipids, which are in fluid-crystalline state, ${ }^{21}$ one can estimate that the CholAU nano-tubules are probably coated by a phospholipid bilayer. In contrast, micro-tubules, which were found to contain about $80 \mathrm{~mol} \%$ of CholAU, ${ }^{21}$ formed only in mixtures with $14: 1,16: 1$ and 18:1 PCs having a lipid bilayer thickness of $\sim 4-5 \mathrm{~nm}^{37,38}$ comparable to the thickness of CholAU bilayers (Fig. S2, ESI $\dagger$ and Fig. 4, 5). This might be an indication that micro-tubules are formed from CholAU/PC mixed bilayers.

Previously, we had reported that phospholipids incorporated into the tubules were in the liquid-crystalline phase state. ${ }^{21}$ Here, we investigated in detail the phase state and molecular dynamics of CholAU using a partially deuterated analog, CholAU- $d_{6}$. The static ${ }^{2} \mathrm{H}$ NMR spectrum of a 60:20:20 mol\% DMoPC/CholAU/ CholAU- $d_{6}$ sample is shown in Fig. 6A.

The ${ }^{2} \mathrm{H}$ NMR spectrum is dominated by a strong isotropic peak indicating that a significant amount of the CholAU- $d_{6}$ is

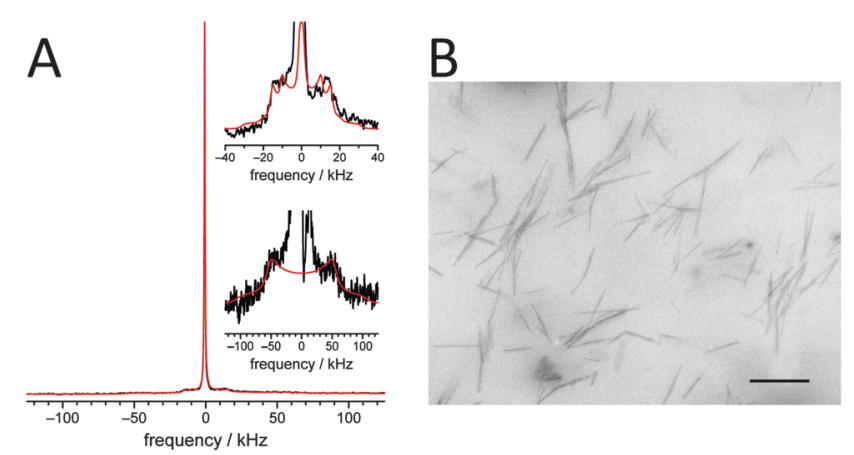

Fig. $6115.1 \mathrm{MHz}$ static ${ }^{2} \mathrm{H}$ NMR spectrum of a $60: 20: 20 \mathrm{~mol} \% \mathrm{DMoPC} /$ CholAU/CholAU- $d_{6}$ sample. (A) The ${ }^{2} H$ NMR spectrum of the sample with a water content of $40 \mathrm{wt} \%$ and at a temperature of $30{ }^{\circ} \mathrm{C}$. The insets show liquid-crystalline (top) and crystalline (bottom) contributions to the enlarged spectrum. Appropriate individual phase corrections had to be applied because of a very large isotropic peak. Red lines show the best fit spectral simulations as described in the text. (B) The fluorescence microscopy image of the probe revealing nano-tubules (inverted). Scale bar corresponds to $10 \mu \mathrm{m}$. 
organized in very small (micelle-like) structures or in structures with high curvatures. In addition, two spectral contributions were detected that show distinct quadrupolar splittings (shown in the insets of Fig. 6A). The NMR spectrum was deconvoluted and the line shapes of the individual components were simulated to determine the individual spectral contributions and to assign them to specific phases present in the sample. ${ }^{60}$ First, we detected an isotropic phase. Spectral simulations revealed that this contribution accounts for about $56 \%$ of the spectral intensity. This means that about half of the CholAU- $d_{6}$ formed isotropic or highly curved phases such as micelles. Second, we detected a liquid-crystalline phase component of the sample, which accounts for about $19 \%$ of the spectral intensity. This phase corresponded to CholAU- $d_{6}$ present in lipid vesicles since cholesterol- $d_{6}$ in vesicles with other lipids showed ${ }^{2} \mathrm{H}$ NMR spectra with quadrupolar splitting on the order of $30-40 \mathrm{kHz} .^{60,61}$ The ratio of the detected quadrupolar splitting in the ${ }^{2} \mathrm{H}$ NMR spectrum of our sample is similar to that determined for cholesterol in liquid-crystalline membranes; ${ }^{61}$ however, the molecular order parameter determined here was 0.51 as opposed to $\sim 0.8$ in previous studies. This can be explained by the higher temperature used in our measurements $\left(30 v s .25{ }^{\circ} \mathrm{C}\right)$ and the fact that the nucleobase moiety of CholAU, which is typically exposed to the aqueous phase ${ }^{62,63}$ and, therefore, may induce some larger amplitude motions of the entire molecule expressed by the somewhat lower order parameter. Finally, we detected a crystalline phase with a large single quadrupolar splitting of $\sim 107 \mathrm{kHz}$, which was indicative of a purely crystalline phase. ${ }^{60}$ This phase accounts for about $25 \%$ of the spectral intensity and can be assigned to the CholAU- $d_{6}$ present in the tubules (Fig. 6B). Similarly, it was suggested before that cholesterol tubules and helices were composed of crystalline cholesterol and covered by a monolayer of phospholipids. ${ }^{19}$

Several mechanisms for tubule formation including rolling up of sheets and growing from helical precursors were proposed before. ${ }^{1,2,64-66}$ In contrast to diacetylenic nano-tubules, which often revealed helical markings, ${ }^{15,49}$ CholAU/PC nano-tubules appeared to be rolled from sheets (Fig. 2, 4 and Fig. S8, S10, ESI $\dagger$ ). Most nano-tubules studied here were completely rolled up into scrolls; however, tubules with the outer sheet partly unwrapped were occasionally observed (Fig. S12, ESI $\dagger$ ). Microtubules resemble furled sheets with loosely rolled morphology; however, helical markings point to the formation through helical ribbons.

To achieve functionalization of the tubules, we first tested spontaneous insertion of a fluorescent lipid analogue into the outer layer of the tubules: both nano- and micro-tubules were labeled with NBD-PC added to the pre-formed unlabeled tubules (Fig. S13A and B, ESI $\dagger$ ). Then we functionalized the tubules with lipophilic nucleic acids with cholesteryl-TEG, tocopherol or palmitoyl anchors that also incorporated spontaneously into the outer layer of the tubular structures; furthermore, the nucleic acids were accessible for the hybridization with fluorescentlylabeled complementary strands (Fig. S13C and D, S14, ESI $\dagger$ ). This paves the way for a further functionalization of the CholAU/ PC tubules. Cross-linking of the tubules by tocopherol-modified
DNA hybrids analogous to the reported cross-linking of lipid vesicles and virus like particles ${ }^{67-69}$ was performed as illustrated in Scheme S4, ESI: $\dagger$ we observed formation of clusters of 3-20 tubules (Fig. S14E and F, ESI $\dagger$ ). Alignment of CholAU/PC tubules by a water flow, for example in microfluidic slides, or by a strong magnetic field ${ }^{21}$ would allow a better cross-linking, e.g. by lipophilic DNA. Recently, nano-particles were interconnected by DNA strands into different crystalline structures. ${ }^{70}$ Spontaneous alignment of lipid nano-tubules at high concentrations ${ }^{71}$ and a directed cell growth in between aligned and plotted on surface nano-carbon tubules was recently reported. ${ }^{72}$ Loading of nano-tubules with a contrast agent and micro-tubules with rhodamine-labeled dextran is illustrated in Fig. S15, ESI. $\dagger$

\section{Conclusions}

Tubular structures self-assembled in aqueous suspensions of cholesterylaminouridine (CholAU) and phosphatidylcholines were investigated using light, electron, and atomic force microscopy and solid-sate NMR. In cryo-SEM samples of the CholAU/PC binary mixtures, we report the same morphologies as observed in aqueous solutions using light microscopy. This proves that the reported protocol of cryo-SEM is applicable to the sensitive lipidbased samples. For the first time, the morphology of the microtubules built from loosely rolled sheets was resolved. In mixtures CholAU with monounsaturated PCs with acyl chain lengths from 14 to 18 carbons, both micro- and nano-tubules formed. In mixtures with saturated PCs or PCs having longer acyl chains only densely packed nano-tubules were observed.

Previously, we reported that the phospholipid coating was in the fluid state. ${ }^{21}$ Here, the ${ }^{2} \mathrm{H}$ NMR analysis on samples containing deuterated CholAU- $d_{6}$ revealed that CholAU in the nano-tubules was in the crystalline state. Fluid and crystalline states are phase separated. The AFM results also indicated the crystalline state of the molecules in the nano-tubules and the flat sheets. $\mathrm{QI}^{\mathrm{TM}}$ AFM allowed determining the thickness of the lamellas in the nano-tubules and the flat structures of about 4-5 $\mathrm{nm}$.

Taken together, our observations suggest that nano-tubules are formed from one or more densely furled sheets, whereas micro-tubules are loosely packed. Our results indicate that the dense nano-tubules, which are extremely enriched in CholAU (up to $92 \mathrm{~mol} \%),{ }^{21}$ have a core of CholAU layers in a crystalline phase with a coating of phospholipids in a liquid crystalline phase. Therefore, the nano-tubules are very stable and rigid, but can be functionalized by lipophilic conjugates, e.g. lipophilic nucleic acids. ${ }^{8,73}$ These tubular structures can be loaded with nutrients or drugs ${ }^{7}$ and cross-linked, e.g. by DNA hybrids, ${ }^{67,68}$ and, therefore, are of interest for further applications, e.g. as a depot-scaffold for tissue regeneration. ${ }^{74,75}$

\section{Conflicts of interest}

The authors declare no competing financial interest. 


\section{Acknowledgements}

This study was supported by the German Research Foundation to AA (AR783/1-1) and EK (SFB740). We are grateful to Prof. J. P. Rabe for advice on and Dr G. Chwastek for help with some AFM measurements, J. Piesker for performing the cryo-ultramicrotomy, and Ch. Grimm for help with some experiments. DH would like to acknowledge support from the graduate school BuildMoNa.

\section{References}

1 T. Shimizu, M. Masuda and H. Minamikawa, Chem. Rev., 2005, 105, 1401-1443.

2 T. G. Barclay, K. Constantopoulos and J. Matisons, Chem. Rev., 2014, 114, 10217-10291.

3 J. M. Schnur, Science, 1993, 262, 1669-1676.

4 A. Bot and W. G. M. Agterof, J. Am. Oil Chem. Soc., 2006, 83, 513-521.

5 J. H. Jung, M. Park and S. Shinkai, Chem. Soc. Rev., 2010, 39, 4286-4302.

6 A.-L. Fameau, F. Cousin, L. Navailles, F. Nallet, F. Boué and J.-P. Douliez, J. Phys. Chem. B, 2011, 115, 9033-9099.

7 W. Ding, M. Wada, H. Minamikawa, N. Kameta, M. Masuda and T. Shimizu, Chem. Commun., 2012, 48, 8625-8627.

8 N. Kameta, H. Minamikawa and M. Masuda, Soft Matter, 2011, 7, 4539-4561.

9 A. L. Fameau, A. Saint-Jalmes, F. Cousin, B. Houinsou Houssou, B. Novales, L. Navailles, F. Nallet, C. Gaillard, F. Boué and J. P. Douliez, Angew. Chem., Int. Ed., 2011, 50, 8264-8269.

10 F. M. Konikoff, D. S. Chung, J. M. Donovan, D. M. Small and M. C. Carey, J. Clin. Invest., 1992, 90, 1155-1160.

11 R. Oda, F. Artzner, M. Laguerre and I. Huc, J. Am. Chem. Soc., 2008, 130, 14705-14712.

12 K. Nagarsekar, M. Ashtikar, J. Thamm, F. Steiniger, F. Schacher, A. Fahr and S. May, Langmuir, 2014, 30, 13143-13151.

13 Y. Qiao, F. Polzer, H. Kirmse, E. Steeg, S. Kühn, S. Friede, S. Kirstein and J. P. Rabe, ACS Nano, 2015, 9, 1552-1560.

14 P. Yager, P. E. Schoen, C. Davies, R. Price and A. Singh, Biophys. J., 1985, 48, 899-906.

15 M. S. Spector, J. V. Selinger, A. Singh, J. M. Rodriguez, R. R. Price and J. M. Schnur, Langmuir, 1998, 14, 3493-3500.

16 M. Gubitosi, L. Travaglini, A. D’Annibale, N. V. Pavel, J. Vázquez Tato, M. Obiols-Rabasa, S. Sennato, U. Olsson, K. Schillén and L. Galantini, Langmuir, 2014, 30, 6358-6366.

17 R. Den Adel, P. C. M. Heussen and A. Bot, J. Phys.: Conf. Ser., 2010, 247, 012025.

18 A. Kaplun, Y. Talmon, F. Konikoff and M. Rubin, FEBS Lett., 1994, 340, 78-82.

19 B. Khaykovich, C. Hossain, J. J. McManus, A. Lomakin, D. E. Moncton and G. B. Benedek, Proc. Natl. Acad. Sci. U. S. A., 2007, 104, 9656-9660.

20 E. Snip, K. Koumoto and S. Shinkai, Tetrahedron, 2002, 58, 8863-8873.
21 P. Pescador, N. Brodersen, H. A. Scheidt, M. Loew, G. Holland, N. Bannert, J. Liebscher, A. Herrmann, D. Huster and A. Arbuzova, Chem. Commun., 2010, 46, 5358-5360.

22 L. Losensky, S. Chiantia, G. Holland, M. Laue, A. Petran, J. Liebscher and A. Arbuzova, RSC Adv., 2015, 5, 4502-4510.

23 J.-P. Douliez, C. Gaillard, L. Navailles and F. Nallet, Langmuir, 2006, 22, 2942-2945.

24 J. H. Georger, A. Singh, R. R. Price, J. M. Schnur, P. Yager and P. E. Schoen, J. Am. Chem. Soc., 1987, 109, 6169-6175.

25 N. Duerr-Auster, J. Kohlbrecher, T. Zuercher, R. Gunde, P. Fischer and E. Windhab, Langmuir, 2007, 23, 12827-12834.

26 A. Jahn, F. Lucas, R. A. Wepf and P. S. Dittrich, Langmuir, 2013, 29, 1717-1723.

27 S. Wiesner, Cryo-SEM images of oil/water emulsions at www.leica-microsystems.com.

28 P. L. Ahl, L. Chen, W. R. Perkins, S. R. Minchey, L. T. Boni, T. F. Taraschi and A. S. Janoff, Biochim. Biophys. Acta, 1994, 1195, 237-244.

29 H. V. Berlepsch, K. Ludwig, B. Schade, R. Haag and C. Böttcher, Adv. Colloid Interface Sci., 2014, 208, 279-292.

30 J. L. M. Leunissen and H. Yi, J. Microsc., 2009, 235, 25-35.

31 J. Bastacky, C. Lee, T. Freeman, G. Weber, A. Baezy, T. Hubbins and Y. Chen, Microsc. Res. Tech., 1995, 32, 457-458.

32 L. Chopinet, C. Formosa, M. P. Rols, R. E. Duval and E. Dague, Micron, 2013, 48, 26-33.

33 J. H. Davis, K. R. Jeffrey, M. Bloom, M. I. Valic and T. P. Higgs, Chem. Phys. Lett., 1976, 42, 390-394.

34 D. Huster, K. Arnold and K. Gawrisch, Biochemistry, 1998, 37, 17299-17308.

35 J. N. Israelachvili, D. J. Mitchell and B. W. Ninham, Biochim. Biophys. Acta, 1977, 470, 185-201.

36 A. D. Bangham and R. W. Horne, J. Mol. Biol., 1964, 8, 660-668.

37 J. Gallová, D. Uhríková, M. Hanulová, J. Teixeira and P. Balgavý, Colloids Surf., B, 2004, 38, 11-14.

38 B. A. Lewis and D. M. Engelman, J. Mol. Biol., 1983, 166, 211-217.

39 S. Milles, T. Meyer, H. A. Scheidt, R. Schwarzer, L. Thomas, M. Marek, L. Szente, R. Bittman, A. Herrmann, T. Günther Pomorski, D. Huster and P. Müller, Biochim. Biophys. Acta, 2013, 1828, 1822-1828.

40 M. Denz, I. Haralampiev, S. Schiller, L. Szente, A. Herrmann, D. Huster and P. Müller, Chem. Phys. Lipids, 2015, DOI: 10.1016/j.chemphyslip.2015.07.017.

41 I. Visco, S. Chiantia and P. Schwille, Langmuir, 2014, 30, 7475-7484.

42 D. J. French, P. Taylor, J. Fowler and P. S. Clegg, J. Colloid Interface Sci., 2015, 441, 30-38.

43 P. Walther, J. Microsc., 2003, 212, 34-43.

44 A. D. Bangham and R. W. Horne, J. Mol. Biol., 1964, 8, 660-668. 45 S. Chiantia, N. Kahya and P. Schwille, Langmuir, 2005, 21, 6317-6323.

46 R. Sorkin, N. Kampf, Y. Dror, E. Shimoni and J. Klein, Biomaterials, 2013, 34, 5465-5475. 
47 Y. Xie, M. Akada, J. P. Hill, Q. Ji, R. Charvet and K. Ariga, Chem. Commun., 2011, 47, 2285-2287.

48 B. N. Thomas, C. R. Safinya, R. J. Plano and N. A. Clark, Science, 1995, 267, 1635-1638.

49 Y. Zhao, L. An and J. Fang, Phys. Rev. E: Stat., Nonlinear, Soft Matter Phys., 2009, 80, 021911.

50 Y. Qiao, Y. Lin, Y. Wang, Z. Yang, J. Liu, J. Zhou, Y. Yan and J. Huang, Nano Lett., 2009, 9, 4500-4504.

51 H. S. Shieh, L. G. Hoard and C. E. Nordman, Nature, 1977, 267, 287-289.

52 R. Abendan and J. Swift, Langmuir, 2002, 11, 4847-4853.

53 J. Marra and J. Israelachvili, Biochemistry, 1985, 24, 4608-4618.

54 J. Pencer, M.-P. Nieh, T. A. Harroun, S. Krueger, C. Adams and J. Katsaras, Biochim. Biophys. Acta, 2005, 1720, 84-91.

55 A. Dols-Perez, L. Fumagalli, A. C. Simonsen and G. Gomila, Langmuir, 2011, 27, 13165-13172.

56 A. Vinckier and G. Semenza, FEBS Lett., 1998, 430, 12-16.

57 J. Huang, J. T. Buboltz and G. W. Feigenson, Biochim. Biophys. Acta, 1999, 1417, 89-100.

58 M. A. Barrett, S. Zheng, L. A. Toppozini, R. J. Alsop, H. Dies, A. Wang, N. Jago, M. Moore and M. C. Rheinstädter, Soft Matter, 2013, 9, 9342-9351.

59 R. Ziblat, I. Fargion, L. Leiserowitz and L. Addadi, Biophys. J., 2012, 103, 255-264.

60 S. Stahlberg, B. Školová, P. K. Madhu, A. Vogel, K. Vávrová and D. Huster, Langmuir, 2015, 31, 4906-4915.

61 E. J. Dufourc, E. J. Parish, S. Chitrakorn and I. C. P. Smith, Biochemistry, 1984, 23, 6062-6071.

62 H. A. Scheidt, W. Flasche, C. Cismas, M. Rost, A. Herrmann, J. Liebscher and D. Huster, J. Phys. Chem. B, 2004, 108, 16279-16287.
63 O. Kaczmarek, H. a. Scheidt, A. Bunge, D. Föse, S. Karsten, A. Arbuzova, D. Huster and J. Liebscher, Eur. J. Org. Chem., 2010, 1579-1586.

64 S. J. Lee, E. Kim, M. L. Seo, Y. Do, Y.-A. Lee, S. S. Lee, J. H. Jung, M. Kogiso and T. Shimizu, Tetrahedron, 2008, 64, 1301-1308.

65 S. A. Morin, M. J. Bierman, J. Tong and S. Jin, Science, 2010, 328, 476-480.

66 Z. Shen, T. Wang and M. Liu, Chem. Commun., 2014, 50, 2096-2099.

67 M. Loew, J. Kang, L. Dähne, R. Hendus-Altenburger, O. Kaczmarek, J. Liebscher, D. Huster, K. Ludwig, C. Böttcher, A. Herrmann and A. Arbuzova, Small, 2009, 5, 320-323.

68 D. Serien, C. Grimm, J. Liebscher, A. Herrmann and A. Arbuzova, New J. Chem., 2014, 38, 5181-5185.

69 U. Jakobsen, A. C. Simonsen and S. Vogel, Nucleic Acids Symp. Ser., 2008, 130, 21-22.

70 B. Nickel and T. Liedl, Nat. Mater., 2015, 14, 746-749.

71 W. Ding, H. Minamikawa, N. Kameta, M. Wada, M. Masuda and T. Shimizu, Langmuir, 2015, 31, 1150-1154.

72 V. Krishnan, Y. Kasuya, Q. Ji, M. Sathish, L. K. Shrestha, S. Ishihara, K. Minami, H. Morita, T. Yamazaki, N. Hanagata, K. Miyazawa, S. Acharya, W. Nakanishi, J. P. Hill and K. Ariga, ACS Appl. Mater. Interfaces, 2015, 7, 15667-15673.

73 M. Schade, D. Berti, D. Huster, A. Herrmann and A. Arbuzova, Adv. Colloid Interface Sci., 2014, 208, 235-251.

74 N. J. Meilander, X. Yu, N. P. Ziats and R. V. Bellamkonda, J. Controlled Release, 2001, 71, 141-152.

75 L. Zarif, J. Controlled Release, 2002, 81, 7-23. 